\title{
How Much Does the Upward Advection of the Supergradient Component of Boundary Layer Wind Contribute to Tropical Cyclone Intensification and Maximum Intensity?
}

\author{
YUANLONG LI \\ Ministry of Education Key Laboratory for Earth System Modeling, Department of Earth System Science, and Joint Center \\ for Global Change Studies, Tsinghua University, Beijing, China, and International Pacific Research Center, and Department \\ of Atmospheric Sciences, School of Ocean and Earth Science and Technology, University of Hawai'i at Mānoa, \\ Honolulu, Hawaii \\ YUQING WANG \\ International Pacific Research Center, and Department of Atmospheric Sciences, School of Ocean and Earth Science \\ and Technology, University of Hawai'i at Mānoa, Honolulu, Hawaii, and State Key Laboratory of Severe Weather, \\ Chinese Academy of Meteorological Sciences, China Meteorological Administration, Beijing, China

\section{YANLUAN LIN} \\ Ministry of Education Key Laboratory for Earth System Modeling, Department of Earth System Science, \\ and Joint Center for Global Change Studies, Tsinghua University, Beijing, China
}

(Manuscript received 23 December 2019, in final form 31 May 2020)

\begin{abstract}
Although the development of supergradient winds is well understood, the importance of supergradient winds in tropical cyclone (TC) intensification is still under debate. One view is that the spinup of the eyewall occurs by the upward advection of high tangential momentum associated with supergradient winds from the boundary layer. The other view argues that the upward advection of supergradient winds by eyewall updrafts results in an outward agradient force, leading to the formation of a shallow outflow layer immediately above the inflow boundary layer. As a result, the spinup of tangential wind in the eyewall by the upward advection of supergradient wind from the boundary layer is largely offset by the spindown of tangential wind due to the outflow resulting from the agradient force. In this study, the net contribution by the upward advection of the supergradient wind component from the boundary layer to the intensification rate and final intensity of a TC are quantified through ensemble sensitivity numerical experiments using an axisymmetric TC model. Results show that consistent with the second view above, the positive upward advection of the supergradient wind component from the boundary layer by eyewall updrafts is largely offset by the negative radial advection due to the outflow resulting from the outward agradient force. As a result, the upward advection of the supergradient wind component contributes little (often less than $4 \%$ ) to the intensification rate and but it contributes about $10 \%-15 \%$ to the final intensity of the simulated TC due to the enhanced inner-core air-sea thermodynamic disequilibrium.
\end{abstract}

\section{Introduction}

Over the past five decades or so, many efforts have been devoted to conceptualizing the physical/dynamical mechanisms responsible for tropical cyclone (TC) intensification. Montgomery and Smith (2014) recently summarized and compared four prominent paradigms of TC intensification in the literature. These are the conditional instability of the second kind (CISK; Charney and

Corresponding author: Yuqing Wang, yuqing@hawaii.edu
Eliassen 1964) paradigm; the cooperative intensification paradigm (Ooyama 1964, 1969, 1982); the wind-induced surface heat exchange (WISHE) feedback paradigm (Rotunno and Emanuel 1987; Emanuel 1989, 1995); and the rotating convective paradigm (Nguyen et al. 2008; Montgomery et al. 2006, 2015; Smith et al. 2009). Each of these paradigms gives, to some extent, a qualitative explanation for the intensification processes of a TC. There are still many remaining scientific mysteries and debates regarding the role of local and nonlocal energy supply, axisymmetric and asymmetric contributions, linear and 
nonlinear processes, relative contributions of balanced and unbalanced dynamics, and so on (e.g., Montgomery and Smith 2017).

More recently, the relative importance/contributions of the balanced and unbalanced dynamics to TC intensification have been under debate (i.e., Bui et al. 2009; Smith et al. 2009; Stern et al. 2015; Smith and Montgomery 2015, 2016; Heng and Wang 2016a,b; Heng et al. 2017, 2018; Montgomery and Smith 2018). The balanced vortex dynamics solves the SawyerEliassen equation to obtain the transverse (secondary) circulation in an axisymmetric vortex in gradient wind balance in response to specified heat and momentum sources (Eliassen 1951). The secondary circulation with its low-level inflow and eyewall updraft in response to diabatic heating in the eyewall transports high absolute angular momentum (AAM) inward to spin up the tangential winds in the inner core of the vortex. The balanced vortex dynamics has been regarded as a classic mechanism of TC intensification with the TC being considered as a "slowly evolving" axisymmetric system (Willoughby 1979; Shapiro and Willoughby 1982; Schubert and Hack 1982; Pendergrass and Willoughby 2009).

The above classic view of TC intensification was challenged by Smith et al. (2009), who proposed that the balanced dynamics in response to eyewall heating spins up the outer circulation of the TC vortex above the boundary layer where the flow is in gradient wind balance and the AAM is conserved following the motion, while the inner-core spinup is largely contributed by the unbalanced dynamics in the boundary layer where the flow is not in gradient balance and the AAM is not conserved due to surface friction. This has been further elaborated later to form the so-called boundary layer spinup mechanism of TC intensification, in which the spinup of supergradient winds is key to the spinup of the inner core of the TC not only in the boundary layer but also above the boundary layer (Smith and Montgomery 2015; Schmidt and Smith 2016; Montgomery and Smith 2017, 2018). By this mechanism, "The spin-up in the boundary layer is associated with the development there of supergradient winds. The spin-up of the eyewall updraught occurs by the vertical advection of the high tangential momentum associated with the supergradient winds in the boundary layer" (Schmidt and Smith 2016, p. 1515; also see Montgomery and Smith 2017, p. 555). Note that the "high tangential momentum" includes both the gradient wind component and the supergradient wind component. The gradient wind component belongs to the balanced dynamics and is determined by the radial gradient of air pressure. The boundary layer spinup mechanism of Montgomery and Smith $(2017,2018)$ emphasizes the unbalanced supergradient wind component as quoted above. This spinup mechanism is considered necessary for TC intensification because in the eyewall updraft above the boundary layer "the flow is outwards (typifying the outward slope of the eyewall) so that the radial advection of absolute angular momentum (or radial flux of absolute vorticity) makes a negative contribution to spinup in this region" (Schmidt and Smith 2016, p. 1515; also see Montgomery and Smith 2017, p. 555). Therefore, Montgomery and Smith (2018, p. 2493) stated that "in an axisymmetric configuration, the spinup of supergradient tangential winds in the boundary layer can provide the necessary negative vertical gradient of $M$ [i.e., absolute angular momentum; our insertion] to spin up the eyewall" above the boundary layer where the flow is outwards.

Note that although the boundary layer spinup hypothesis emphasizes the supergradient wind component, their results did not split the supergradient wind component from the total upward advection (e.g., Schmidt and Smith 2016). One issue regarding the boundary layer spinup mechanism, therefore, has not been addressed, namely, whether the upward advection of the supergradient wind component from the boundary layer dominates the spinup of tangential wind in the eyewall above the boundary layer. Based on a TC boundary layer model, Kepert and Wang (2001) showed that the outflow (typifying the outward slope of the eyewall) immediately above the boundary layer inflow develops in response to supergradient momentum carried aloft by the eyewall updraft. This led Heng et al. (2018) to hypothesize that the spinup of tangential wind in the eyewall due to upward advection of supergradient winds from the boundary layer could be largely offset by the spindown due to the outflow resulting from the outward agradient force due to the upward advection of supergradient winds. Therefore, they argued that the upward advection of the supergradient wind component from the boundary layer might not be a dominant mechanism for the overall TC intensification.

This study attempts to quantify the degree to which the upward advection of the supergradient wind component from the boundary layer contributes to the TC intensification rate and maximum intensity of a numerically simulated TC based on ensemble simulations using an axisymmetric convection-permitting TC model. Note that the axisymmetric model is used here, as in Schmidt and Smith (2016), because the boundary layer spinup mechanism is also introduced based on the axisymmetric argument. We will show that the boundary layer spinup mechanism related to the upward advection of the supergradient wind component from the boundary layer contributes little (often less than $4 \%$ ) to TC 
TABLE 1. List of numerical experiments.

\begin{tabular}{lll}
\hline \hline \multicolumn{1}{c}{ Expt } & \multicolumn{1}{c}{$C_{d}$} & \multicolumn{1}{c}{ Vertical advection of tangential wind } \\
\hline CTL & $2.58 \times 10^{-3}$ & $-w \partial v / \partial z$ \\
Vg3 & $2.58 \times 10^{-3}$ & $-w \partial v / \partial z$ but $-w \partial \min \left(v, v_{g}\right) / \partial z$ if $r \leq 50 \mathrm{~km}, z \leq 3 \mathrm{~km}, t \geq 24 \mathrm{~h}$, and $-w \partial v / \partial z>0$ \\
Vg4 & $2.58 \times 10^{-3}$ & $-w \partial v / \partial z$ but $-w \partial \min \left(v, v_{g}\right) / \partial z$ if $r \leq 50 \mathrm{~km}, z \leq 4 \mathrm{~km}, t \geq 24 \mathrm{~h}$, and $-w \partial v / \partial z>0$ \\
$05 C d$ & $1.29 \times 10^{-3}$ & $-w \partial v / \partial z$ \\
05Cd_Vg3 & $1.29 \times 10^{-3}$ & $-w \partial v / \partial z$ but $-w \partial \min \left(v, v_{g}\right) / \partial z$ if $r \leq 50 \mathrm{~km}, z \leq 3 \mathrm{~km}, t \geq 27 \mathrm{~h}$, and $-w \partial v / \partial z>0$ \\
15Cd & $3.87 \times 10^{-3}$ & $-w \partial v / \partial z$ \\
15Cd_Vg3 & $3.87 \times 10^{-3}$ & $-w \partial v / \partial z$ but $-w \partial \min \left(v, v_{g}\right) / \partial z$ if $r \leq 50 \mathrm{~km}, z \leq 3 \mathrm{~km}, t \geq 21 \mathrm{~h}$, and $-w \partial v / \partial z>0$ \\
20Cd & $5.16 \times 10^{-3}$ & $-w \partial v / \partial z$ \\
20Cd_Vg3 & $5.16 \times 10^{-3}$ & $-w \partial v / \partial z$ but $-w \partial \min \left(v, v_{g}\right) / \partial z$ if $r \leq 50 \mathrm{~km}, z \leq 3 \mathrm{~km}, t \geq 21 \mathrm{~h}$, and $-w \partial v / \partial z>0$ \\
\hline
\end{tabular}

intensification and thus should not be considered as a dominant mechanism of TC intensification, but contributes positively to the final intensity by about $10 \%-15 \%$. The rest of the paper is organized as follows. The model and experimental designs are described in section 2 . Results from the control ensemble experiment and the sensitivity ensemble experiments with the upward advection of supergradient winds suppressed are discussed in section 3. The sensitivity of the main results from section 3 to surface drag coefficient is examined in section 4. Our major findings are summarized and discussed in the last section.

\section{Model and experimental design}

The axisymmetric model used in this study is the stateof-the-art Cloud Model 1 (CM1), version 19.8 (Bryan and Fritsch 2002). The domain size is $3100 \mathrm{~km} \times 25 \mathrm{~km}$. The radial resolution within $100-\mathrm{km}$ radius is $1 \mathrm{~km}$ and is stretched to $12 \mathrm{~km}$ at the outer boundary. The model has 59 vertical levels with stretched grids below $5.5 \mathrm{~km}$ as in Li et al. (2019). The moist tropical sounding of Dunion (2011) is used as the unperturbed environment of the initial condition. The sea surface temperature is set constant at $29^{\circ} \mathrm{C}$. An $f$ plane is assumed with the Coriolis parameter set to $5 \times 10^{-5} \mathrm{~s}^{-1}$. Similar to Montgomery et al. (2015), a warm rain microphysics scheme (Kessler 1969) is used for cloud/precipitation processes and no cumulus convective parameterization is used in all simulations. Newtonian cooling, capped at $2 \mathrm{~K} \mathrm{day}^{-1}$, is added to the thermodynamic equation to mimic radiative cooling (Rotunno and Emanuel 1987), while dissipative heating is not included for simplicity. As in Montgomery et al. (2015), the ratio of surface enthalpy exchange coefficient to surface drag coefficient is set at $C_{k} / C_{D}=0.5$ with surface drag coefficient $C_{D}$ being $2.58 \times 10^{-3}$. The subgrid-scale turbulent mixing is parameterized using the Smagorinsky scheme (Bryan and Fritsch 2002), and the corresponding horizontal and asymptotic vertical mixing lengths are fixed at 700 and $50 \mathrm{~m}$, respectively, also the same as those used in Montgomery et al. (2015).

The initial TC vortex has a radial profile of tangential wind speed following Wood and White (2011). The initial maximum tangential wind speed is $15 \mathrm{~m} \mathrm{~s}^{-1}$ at $80-\mathrm{km}$ radius in the standard run of each ensemble experiment. The radial shape parameter is set to be 1.6. The tangential wind speed decreases linearly with height to zero at $18-\mathrm{km}$ height. Each ensemble experiment has 21 members. In addition to the standard run, each of the remaining 20 members are generated by perturbing the initial radius of maximum wind (RMW) by an increment of $\pm 0.4 \mathrm{~km}$ (for 10 runs) or the initial maximum wind speed by $\pm 0.1 \mathrm{~m} \mathrm{~s}^{-1}$ (for 10 runs). All 21 runs for each experiment (see description below) are integrated for $120 \mathrm{~h}$ with the model output saved at every $6 \mathrm{~min}$ for the purpose of composite and budget analyses. The ensemble experiments are designed to remove internal variability and make sure of the robustness of the results from sensitivity simulations. Only the ensemble composite from each experiment is discussed in this study. Note that our preliminary tests indicate that the results discussed herein are insensitive to the perturbation increments within reasonable ranges.

To address whether and to what extent the upward advection of supergradient winds from the boundary layer contributes to the overall intensification rate and final intensity of a TC, two ensemble experiments are conducted (Table 1). In the control experiment (labeled by CTL), the model is run with all default settings as described above. In the sensitivity experiment, the vertical advection of tangential winds in the inner core region is modified in each run so that the upward advection of the supergradient wind component in the tangential momentum equation is omitted. Note that only the positive (upward) vertical advection is modified, so that the boundary layer spinup mechanism as articulated by Schmidt and Smith (2016) and Montgomery and Smith (2017) as mentioned in section 1 is suppressed. Specifically, the positive vertical advection term $-w \partial v / \partial z$ in the tangential momentum 
equation is replaced by $-w \partial\left[\min \left(v, v_{g}\right)\right] / \partial z$, where $z$ denotes height, and $w, v$, and $v_{g}$ denote vertical velocity, tangential wind speed, and gradient wind speed, respectively. The gradient wind $v_{g}$ in CM1 is calculated as follows:

$$
v_{g}=-\frac{f r}{2}+\left(\frac{f^{2} r^{2}}{4}+r c_{p} \theta_{v} \frac{\partial \pi^{\prime}}{\partial r}\right)^{1 / 2}
$$

where $r$ is the radius, $c_{p}$ the specific heat of dry air at constant pressure, and $\theta_{v}$ and $\pi^{\prime}$ are virtual potential temperature and nondimensional pressure, respectively. Figure 1 shows an example of the modified vertical advection (Fig. 1c) from the unmodified vertical advection (Fig. 1a) using the model output after $48 \mathrm{~h}$ of simulation from CTL. As we can see from Fig. 1c, by the approach used in our sensitivity experiment, the upward (positive) advection of supergradient winds from the boundary layer (Fig. 1b), namely, the dominant process contributing to the eyewall spinup as articulated in the boundary layer spinup mechanism of the eyewall by Schmidt and Smith (2016) and Montgomery and Smith (2017), is clearly omitted. Note that because our focus is on the upward advection of the supergradient wind component, as emphasized by the boundary layer spinup hypothesis, rather than the supergradient wind itself, we allow the supergradient wind to develop but only suppress the upward advection of the supergradient wind component. Note also that we do not mean the sensitivity experiment to be "realistic simulation," rather it is a thought experiment that is designed to allow the above mentioned process to be quantified.

Because we focus on the intensification process of the simulated TC, the replacement of the vertical advection term in all sensitivity members is activated after the initial 24-h adjustment (cf. Fig. 2a). By this time, the RMW reaches $\sim 45 \mathrm{~km}$ and contracts continuously from then on (cf. Fig. 2b). Therefore, the replacement of the vertical advection term is confined to the inner core region within a radius of $50 \mathrm{~km}$ (Table 1 ). In addition, given the fact that supergradient winds are mainly located in the boundary layer and below $\sim 2-3 \mathrm{~km}$ (cf. Fig. 3), the replacement of vertical advection is confined at low levels below $3 \mathrm{~km}$ height (labeled by $\mathrm{Vg} 3$, Table 1). To ensure the robustness of the main results, the sensitivity experiment is repeated with the replacement of vertical advection confined below $4 \mathrm{~km}$ height (labeled by $\mathrm{Vg} 4$ ).

Considering the fact that supergradient winds result from surface friction and may change with surface drag coefficient, we conduct six extra ensemble experiments using different surface drag coefficients to ensure the robustness of the results. The surface drag coefficient in
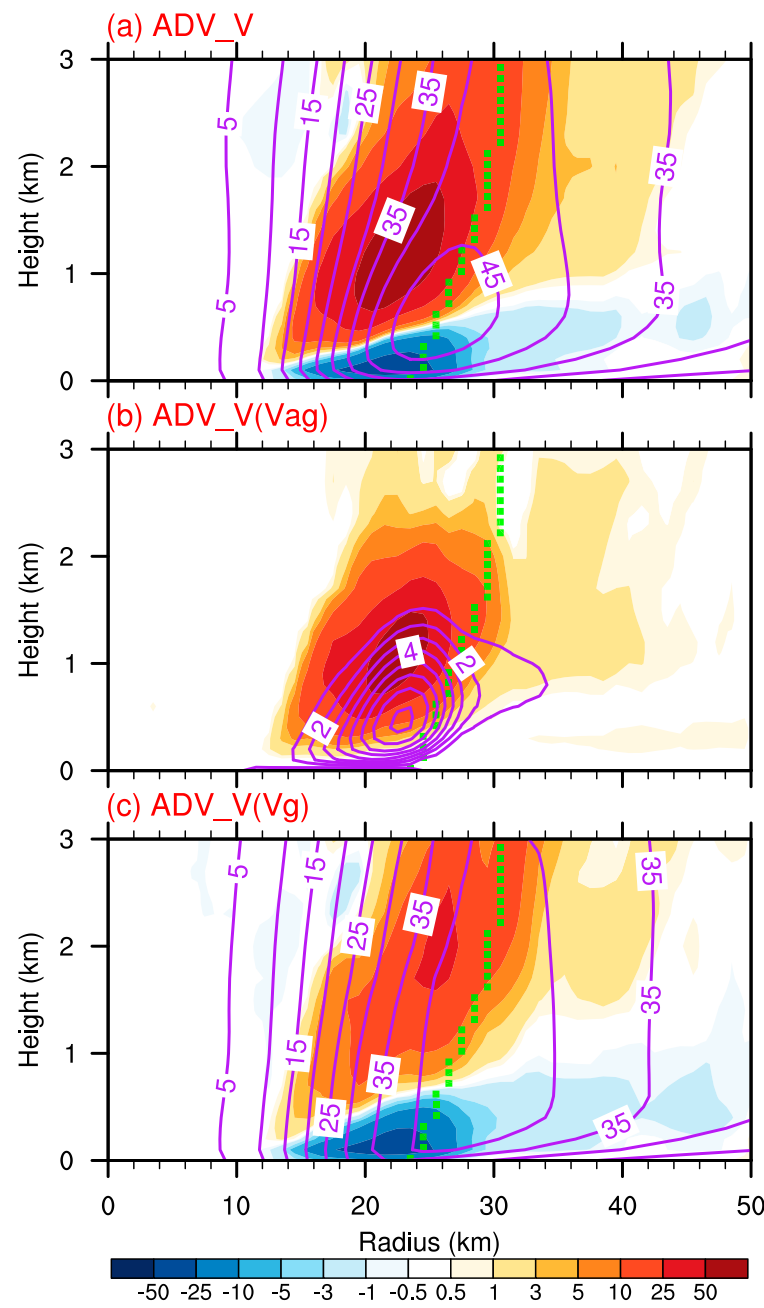

FIG. 1. (a) The radial-vertical cross sections of the ensemblemean tangential wind speed ( $v$, purple contours; $\mathrm{m} \mathrm{s}^{-1}$ ) and vertical advection of total tangential wind (ADV_V, shading; $\mathrm{m} \mathrm{s}^{-1} \mathrm{~h}^{-1}$ ) at $48 \mathrm{~h}$ of simulation in CTL. (b) As in (a), but for the supergradient winds $\left[\operatorname{Vag}=v-\min \left(v, v_{\mathrm{g}}\right)\right]$ and the corresponding vertical advection $\left[\mathrm{ADV}_{-} \mathrm{V}(\mathrm{Vag})\right]$. (c) $\operatorname{Min}\left(v, v_{g}\right)$ (contours) and the difference between the vertical advection of total tangential wind (shading) as given in (a) and the vertical advection of supergradient winds as given in (b) [ADV_V(Vg)]. Note that each advection in (a)-(c) is diagnosed using the same finite-difference scheme in CM1 and in each individual run. Note that in order to modify the positive upward advection related to the supergradient wind component only, ADV_V(Vag) is set to zero and ADV_V $(\mathrm{Vg})$ is set to $A D V_{-} V$ if the ADV_V is negative. The dotted green line shows the location of the RMW at each level.

the CTL experiment described above is multiplied by $0.5,1.5$, and 2.0 , respectively, in experiments $05 \mathrm{Cd}$, $15 \mathrm{Cd}$, and $20 \mathrm{Cd}$, with all default model settings as in the CTL experiment. In three other sensitivity experiments $\left(05 \mathrm{Cd} \_\mathrm{Vg} 3,15 \mathrm{Cd} \_\mathrm{Vg} 3\right.$, and $\left.20 \mathrm{Cd} \_\mathrm{Vg} 3\right)$, the positive vertical advection of the supergradient wind component within a $50-\mathrm{km}$ radius and below $3 \mathrm{~km}$ is omitted 

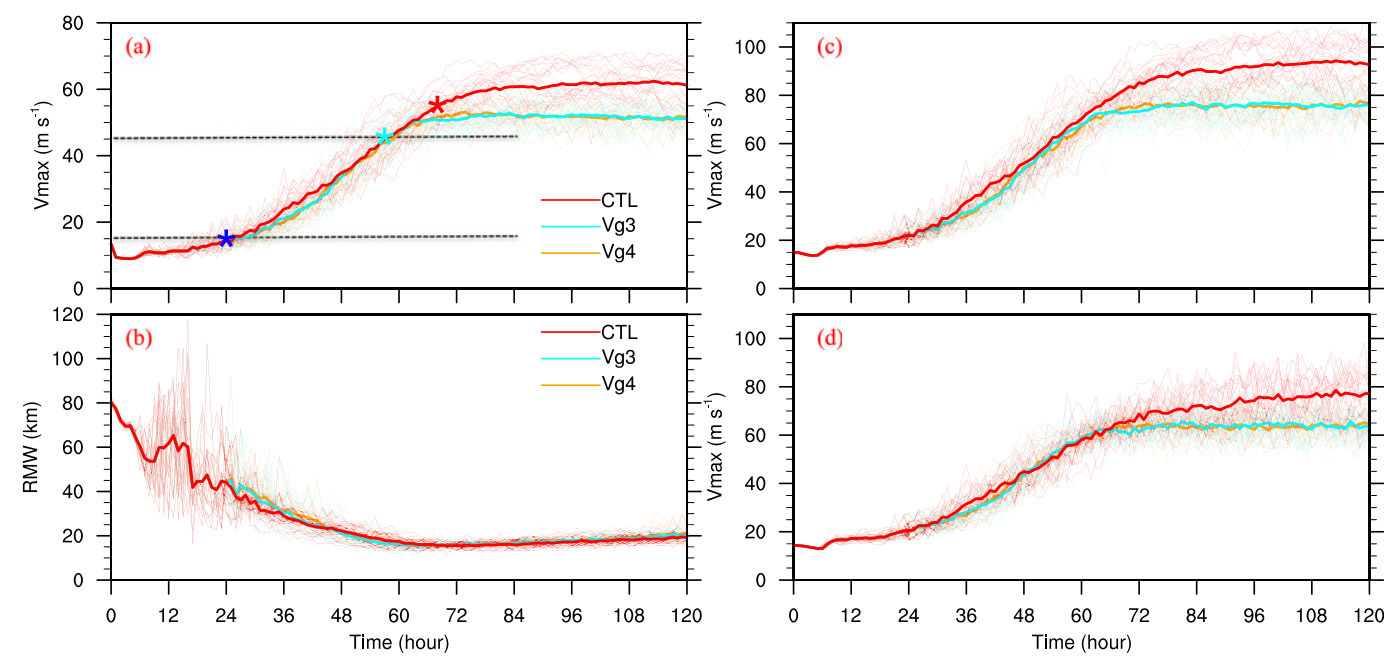

FIG. 2. Time series of (a) the maximum 10-m tangential wind speed and (b) the radius of maximum 10-m tangential wind speed from experiments CTL, Vg3, and $\mathrm{Vg} 4$. In (c) and (d), time series of the maximum tangential wind anywhere in the interior of the boundary layer and that at 2-km height are shown. Results from the 21 individual members and the ensemble mean for each experiment are shown in thin and thick curves. The two dashed black horizontal lines in (a) mark the period for the average radial-vertical cross sections shown in Figs. 4-6. The blue asterisk in (a) marks the approximate onset of the primary intensification stage of all three experiments, and the red and cyan asterisks mark the end of the primary intensification stage in CTL and Vg3.

as in experiment $\mathrm{Vg}$. Note that because the duration of the initial adjustment varies slightly with surface drag coefficient, the omission of the vertical advection of the supergradient wind component is activated after $27 \mathrm{~h}$ run of $05 \mathrm{Cd}$ in $05 \mathrm{Cd} / \mathrm{Vg} 3,21 \mathrm{~h}$ run of $15 \mathrm{Cd}$ in $15 \mathrm{Cd} \_\mathrm{Vg} 3$, and $21 \mathrm{~h}$ run of $20 \mathrm{Cd}$ in $20 \mathrm{Cd} \_\mathrm{Vg} 3$ (Table 1) by subjectively chosen. Note that the results discussed below are not affected by the time and the space of the replacement of vertical advection in the sensitivity experiments. This is because that the supergradient wind and its upward advection are very marginal during the early stage of TC intensification or outside the eyewall (cf. Fig. 3)

\section{Contributions of upward advection of supergradient winds}

Figures $2 \mathrm{a}$ and $2 \mathrm{~b}$ compare the time series of maximum 10-m height wind speed (TC intensity) and the corresponding RMW from experiments CTL, $\mathrm{Vg} 3$, and $\mathrm{Vg} 4$, respectively. Consistent with the hypothesis in the recent literature (Smith and Montgomery 2015; Schmidt and Smith 2016; Montgomery and Smith 2017, 2018), the upward advection of supergradient winds contributes positively to TC intensification during the early intensification period and quasi-steady intensity (Fig. 2a). However, consistent with the hypothesis of Heng et al. (2018), but in contrast to the boundary layer spinup mechanism proposed by Schmidt and Smith (2016) and
Montgomery and Smith $(2017,2018)$, this contributes little $(<1 \%)$ to the intensification rate during the primary intensification stage, but it contributes $\sim 15 \%$ of the final quasi-steady intensity of the simulated TC. Note that the "primary intensification stage" here is defined as a continuous period within which the TC intensity increased by $5 \mathrm{~m} \mathrm{~s}^{-1}$ or more in the following $12 \mathrm{~h}$ after the onset of the intensification (see asterisks marked in Fig. 2a). The effect of upward advection of supergradient winds on both the contraction of the RMW at 10-m height and the final RMW are minor (Fig. 2b). In addition to the maximum tangential wind at $10-\mathrm{m}$ height, we also compared the maximum tangential winds anywhere in the interior of the boundary layer (Fig. 2c) and that at 2-km height (Fig. 2d). Overall, the results are consistent with those discussed above although the maximum tangential wind in the interior of the boundary layer is considerably greater than that at $10-\mathrm{m}$ height or at $2-\mathrm{km}$ height because of the large supergradient nature of tangential wind in the boundary layer and subgradient nature near the surface. From Fig. 2, we also can see that the results from $\mathrm{Vg} 3$ and $\mathrm{Vg} 4$ are quite similar, confirming that the upward advection of supergradient winds mainly occurred below 3-km height in the simulation (cf. Fig. 3). Therefore, we will mainly focus on the results from $\mathrm{Vg} 3$ in the following discussion.

The above results strongly suggest that the upward advection of the supergradient wind component is not a 

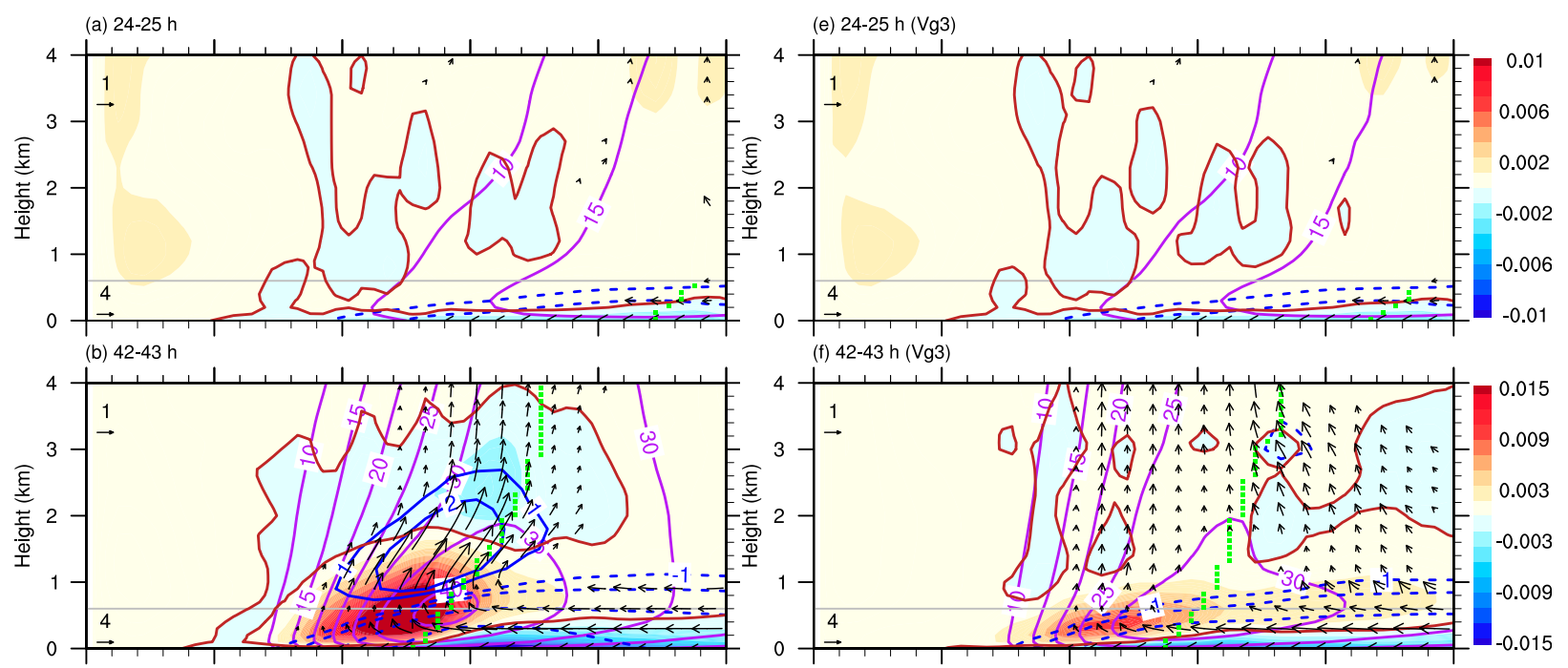

(f) $42-43 \mathrm{~h} \mathrm{(Vg3)}$
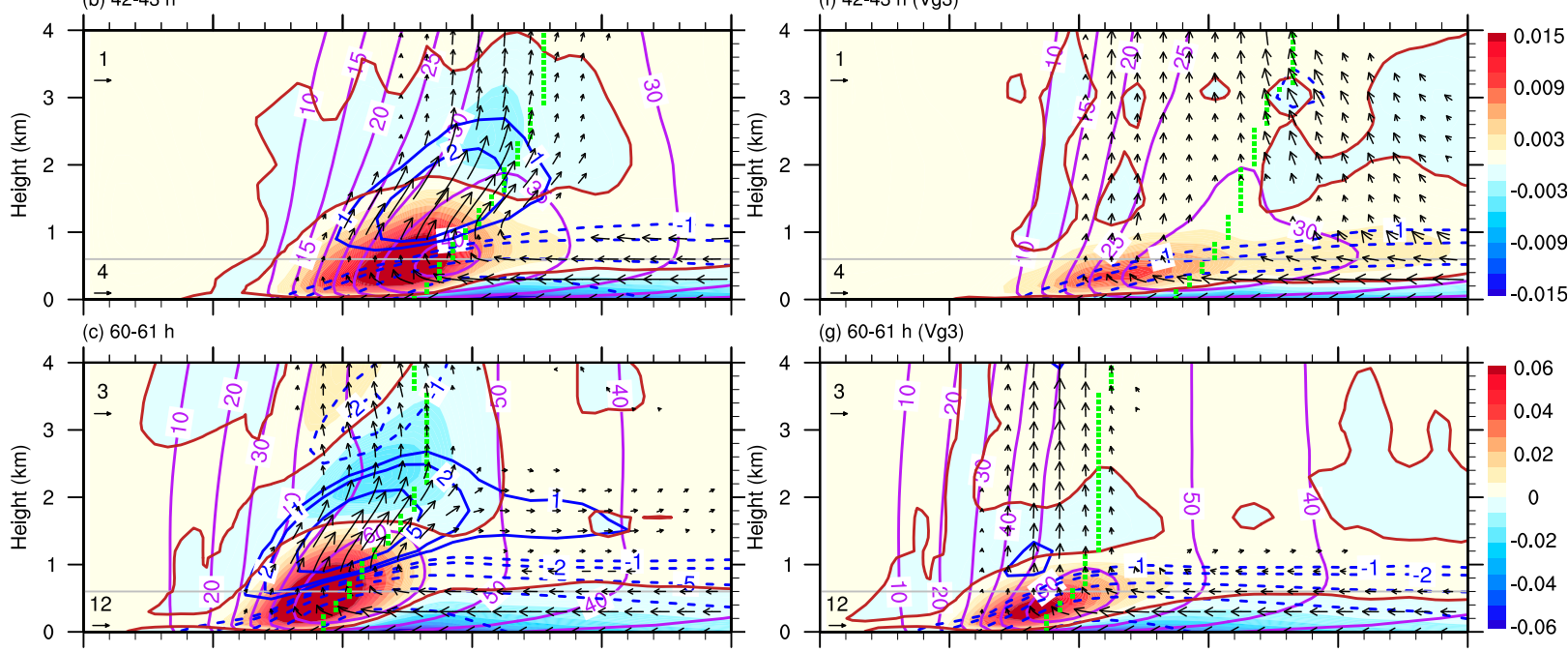

(g) $60-61 \mathrm{~h} \mathrm{(Vg3)}$
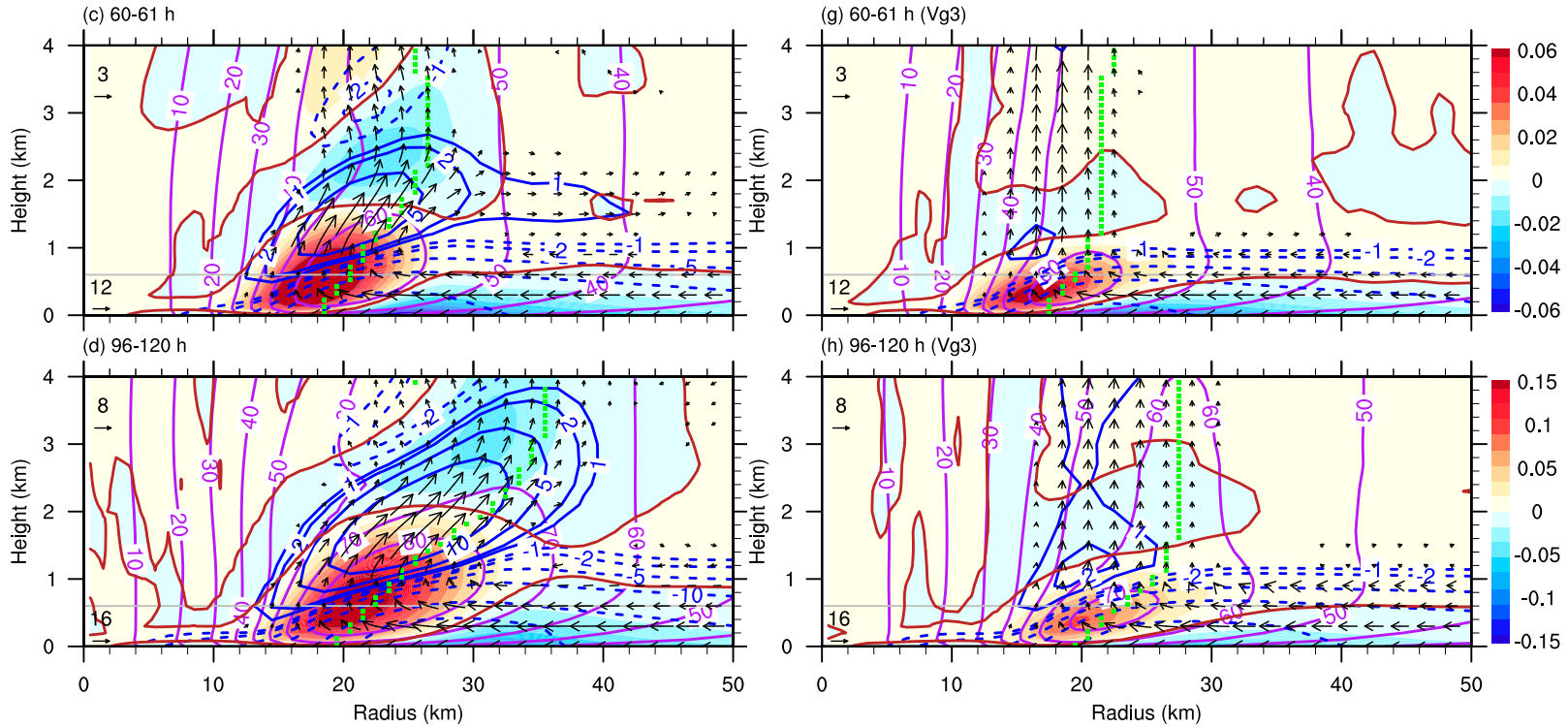

FIG. 3. The radial-vertical cross sections of the ensemble-mean agradient force (shading with zero contour highlighted in brown; $\mathrm{m} \mathrm{s}^{-2}$ ), tangential wind speed (purple contours; $\mathrm{m} \mathrm{s}^{-1}$ ), radial wind (blue contours with negative values dashed; $\mathrm{m} \mathrm{s}^{-1}$ ), and the transverse circulation (black vectors, only with the total wind speed greater than $0.3 \mathrm{~m} \mathrm{~s}^{-1}$ shown, note that different reference magnitudes are used below and above $0.6-\mathrm{km}$ heights marked by the gray horizontal line) averaged between (a) 24-25, (b) 42-43, (c) 60-61, and (d) 96-120 h using model outputs at 6-min intervals from CTL. (e)-(h) As in (a)-(d), but from Vg3. The dotted green line shows the radial location of the RMW at each level. Note that the scale of color bar in each row is different.

dominant mechanism of TC intensification, such as the spinup of the eyewall as hypothesized in some previous studies (Schmidt and Smith 2016; Montgomery and Smith 2017, 2018). To understand why the upward advection of supergradient winds from the boundary layer is not crucial to TC intensification, we first compare in Fig. 3 the evolution of the ensemble mean boundary layer structures in CTL and Vg3. At the beginning of intensification (Fig. 3a), the agradient force $\left(=f v+v^{2} / r-c_{p} \theta_{v} \partial \pi^{\prime} / \partial r\right)$ in the inner-core region is generally small, and thus there is no obvious supergradient wind (not shown, which has the same spatial pattern as the agradient force by definition) in the boundary layer. Therefore, the differences in TC intensity (Fig. 2a) and the boundary layer structure (Figs. 3a,e) between CTL and $\mathrm{Vg} 3$ are negligible in the early stage of intensification, also partly because this is the first hour after the imposition of the modified advection. However, later on, as the TC intensifies, the inward agradient force appears in the surface layer near and outside the RMW due to surface friction, leading to the development of strong inflow near the surface in both CTL and Vg3. A 
local maximum in outward agradient force, which results from the amplification of supergradient winds as the storm intensifies, appears above the surface layer inside the RMW in CTL (Figs. 3b-d). The outward agradient force in the boundary layer leads to the development of an outflow layer in the upper part of the inflow boundary layer and in the lower troposphere in CTL (Figs. 3b-d). In the upper part of the outflow layer, a local maximum in inward agradient force appears, reflecting the existence of subgradient winds (Figs. 3b-d). This alternative appearance of supergradient and subgradient winds is consistent with the vertical oscillation of the AAM surfaces discussed in Rotunno and Bryan (2012), and has been proven to be a common structure in rotating-flow boundary layers (Rotunno 2014).

With the positive vertical advection of the supergradient wind component removed in $\mathrm{Vg} 3$, the amplitude of oscillation of the AAM surfaces, supergradient winds and the corresponding outflow layer in the upper part of the boundary layer (Figs. $3 \mathrm{f}-\mathrm{h}$ ) are largely reduced compared with those in CTL (Figs. 3b-d), indicating that the vertical advection of supergradient winds largely contributes to the enhancement of supergradient winds in the boundary layer and outflow aloft as demonstrated by Kepert and Wang (2001). Since the outflow above the frictional boundary layer often causes the spindown of tangential wind, Heng et al. (2018) speculated that the spinup of tangential wind resulting from the upward advection of supergradient winds might be largely offset by the spindown due to the forced outflow. As a result, the net effect of the upward advection of supergradient winds from the boundary layer should not be crucial to TC intensification.

In addition to the reduction of the outward agradient force in the upper part of and immediately above the inflow boundary layer, the inward agradient force in the lower part of the inflow boundary layer also becomes weaker in the inner-core region in $\mathrm{Vg} 3$ than in CTL. Because of the reduction of supergradient winds aloft, the near-surface negative upward advection of tangential wind, which decelerates the local tangential wind and enhances the near-surface inward agradient force, would decrease. As a result, the reduced inward agradient force under the eyewall in $\mathrm{Vg} 3$ leads to reduced boundary layer inflow (Figs. 3f-h), and meantime, the inflow and inward agradient force outside the eyewall (near the layer of peak inflow) are also reduced (Figs. 3f-h). This suggests that the increase in inner-core radial advection of tangential wind due to enhanced inflow in association with the spinup of supergradient wind is offset by the increase of negative vertical advection of tangential wind in the presence of supergradient wind. This will be confirmed by results from tangential wind budget analyses discussed below.

To understand why the upward advection of supergradient winds contributes little to the overall TC intensification rate, we further examine the tangential wind budgets for the simulated TCs in CTL and Vg3 during their primary intensification stages (Figs. 4-6 ). The tangential wind tendency equation in the axisymmetric version of CM1 can be written as (Li et al. 2019)

$$
\frac{\partial v}{\partial t}=-u \xi_{a}-w \frac{\partial v}{\partial z}+F_{h}+F_{v}
$$

where $u$ denotes radial wind speed, $\xi_{a}=\partial v / \partial r+v / r+f$ denotes absolute vertical vorticity. The term on the lhs of Eq. (2) is the net local tangential wind tendency (NET), and the four terms on the rhs are radial advection $\left(A D V \_H\right)$, vertical advection $\left(A D V_{-}\right.$V), turbulent horizontal mixing (DIFF_H), and turbulent vertical mixing including surface friction (DIFF_V). As in Li et al. (2019), all instantaneous terms in Eq. (2), including the NET, are output directly from the model simulations at a 6-min interval. Therefore, the budget is residual free. We compare the tangential wind budgets in CTL and $\mathrm{Vg} 3$ during their corresponding primary intensification stages. The budgets are averaged in the period during which the storms in CTL and Vg3 have the same ensemble-mean 10-m tangential wind speed, i.e., between 15 and $45 \mathrm{~m} \mathrm{~s}^{-1}$ (the two black dashed horizontal lines in Fig. 2a). Note that although some small shift of RMW ( $\leq 2 \mathrm{~km}$; Fig. 6 ) occurs between the two experiments, the overall budget results are insensitive to the period chosen for the time averaging (not shown).

In CTL, large positive tangential wind tendencies in the inflow boundary layer reflect the inward transport of AAM by the boundary layer inflow while a relatively deep layer of negative tendencies inside the RMW immediately above the positive tendencies results from the outflow (Fig. 4a) associated with the outward agradient force as discussed above. Vertical advection due to upward motion in the eyewall induces negative tangential wind tendencies in the lower part of the inflow boundary layer and large positive tendencies immediately above (Fig. 4b). This is mainly because the supergradient wind peaks in the interior of the inflow boundary layer, giving rise to a positive vertical gradient of tangential wind below and a negative vertical gradient above. Although the negative tangential wind tendencies induced by vertical advection in the lower part of the inflow boundary layer inside the RMW is smaller compared with the positive tendencies induced by the radial advection, the positive tendencies induced by vertical advection above the inflow boundary layer are largely offset by the negative tendencies induced by the 

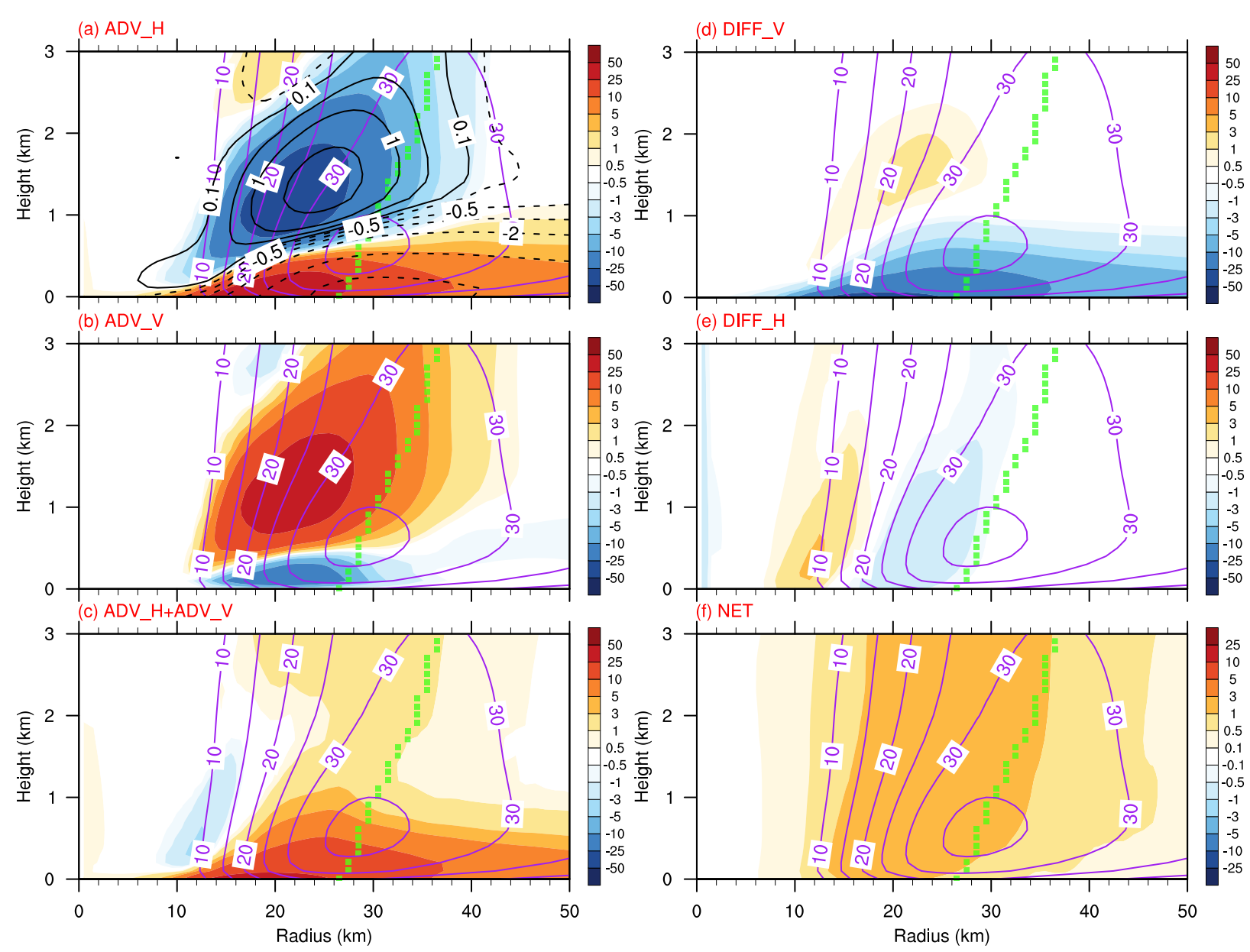

FIG. 4. The radial-vertical cross sections of the ensemble-mean tangential wind speed (purple contours; $\mathrm{m} \mathrm{s}^{-1}$ ) and tangential wind tendencies (shading; $\mathrm{m} \mathrm{s}^{-1} \mathrm{~h}^{-1}$ ) averaged between the two dashed horizontal lines in Fig. 2a from CTL due to (a) radial advection, (b) vertical advection, (c) total advection, (d) vertical mixing including friction, (e) horizontal mixing, and (f) net budget. The radial wind $\left(\mathrm{m} \mathrm{s}^{-1}\right)$ is also shown in black contours with negative values dashed in (a). The dotted green lines show the radial location of the RMW. Note that the label bar for the net budget in (f) is different from other terms.

radial advection, resulting in relatively weak positive tendencies (Fig. 4c). The positive tendencies in the inflow boundary layer by total advection (Fig. 4c) is largely compensated by the negative tendencies due to vertical mixing including surface friction (Fig. 4d). Horizontal diffusion results in some small negative tangential wind tendencies inside the RMW and some small positive tendencies farther inside in the eye region (Fig. 4e). As a result, the net tangential wind tendencies (NET) shown in Fig. 4f is consistent with the intensification of the simulated storm. Note that the error between the NET (the sum of all terms from the 6-min model output) and the actual tangential wind tendencies is small (not shown).

The removal of vertical advection of the supergradient wind component in Vg3 leads to a substantial reduction of both negative tangential wind tendencies induced by radial advection and positive tendencies induced by vertical advection immediately above the inflow boundary layer (Figs. 5a,b). As a result, the total advection-induced positive tangential wind tendencies inside the RMW above the inflow boundary layer in $\mathrm{Vg} 3$ (Fig. 5c) are quite similar to those in CTL (Fig. 4c). This means that although the vertical and radial advections are largely offsetting terms (Figs. $4 a-c$ ) for both the supergradient flow and the gradient flow, the cancellation is more nearly complete for the supergradient flow and it is the vertical advection of the gradient flow that is largely responsible for spinning up the eyewall above the boundary layer (Figs. 5a-c). In addition, the vertical gradient of tangential winds in the lower part of the inflow boundary layer is reduced in $\mathrm{Vg} 3$ because of the reduction of supergradient winds (Fig. 3) in response to the removal of the upward advection of the supergradient 

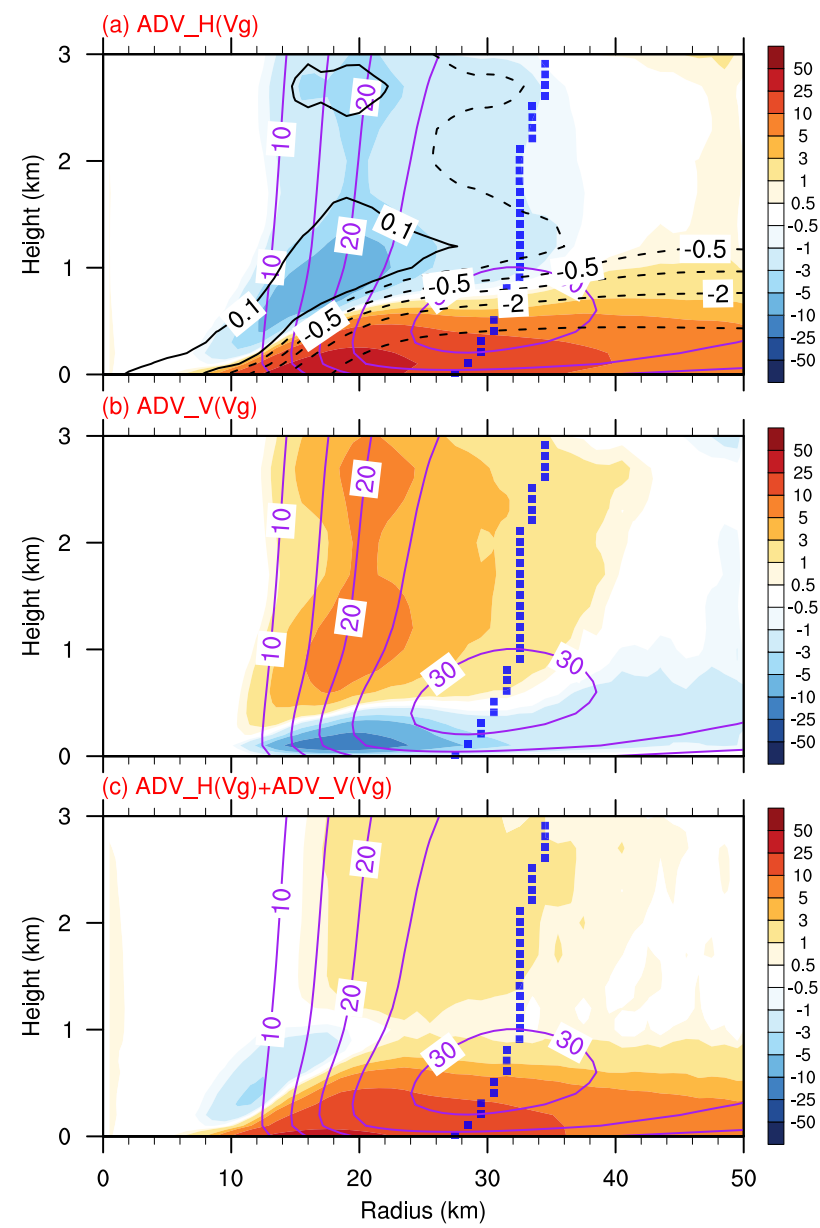

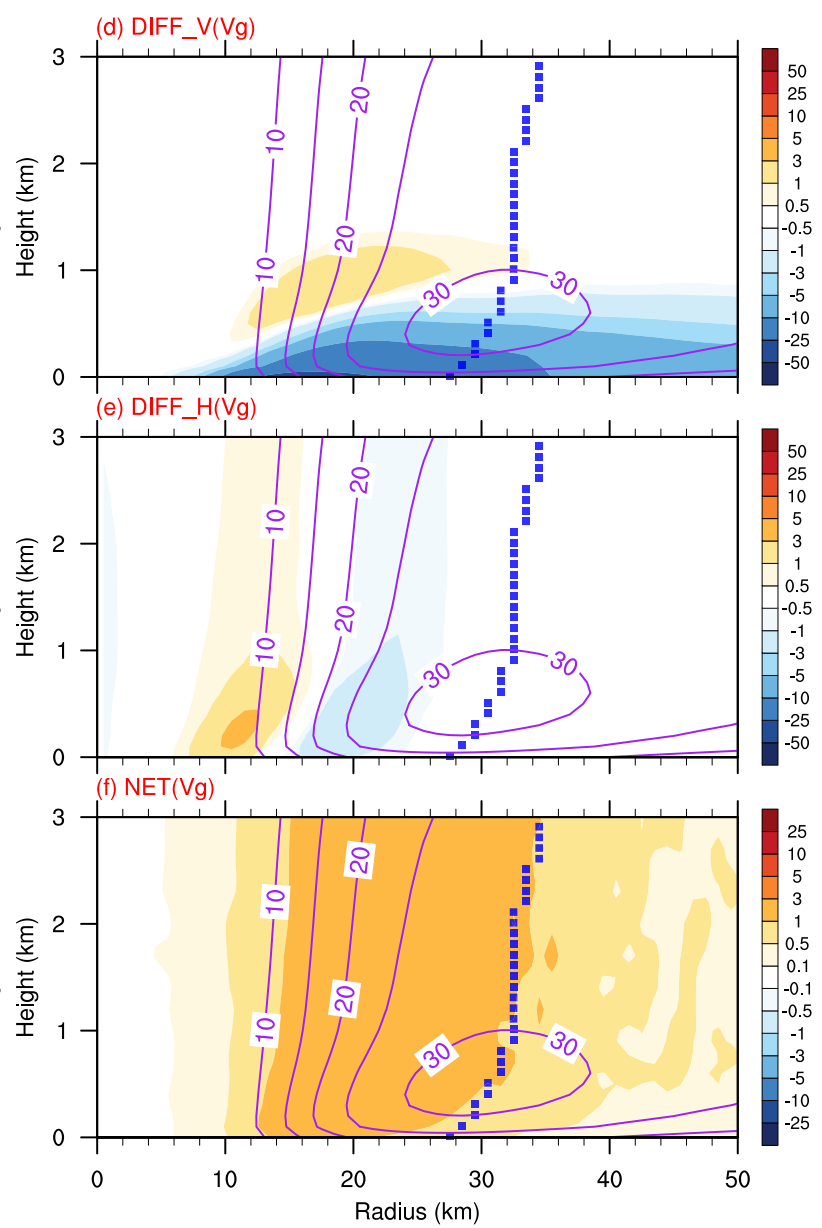

FIG. 5. As in Fig. 4, but for the results from Vg3 and the radial location of the RMW is shown by dotted blue lines.

wind component as mentioned above. This leads to a reduction of the negative tangential wind tendencies due to vertical advection in the lower part of the inflow boundary layer (Fig. 5b) compared to those in CTL (Fig. 4b). This reduction is largely compensated by the reduction of positive tendencies contributed by radial advection (Fig. 5a) due to the reduced inflow in the lower part of the inflow boundary layer inside the RMW in $\mathrm{Vg} 3$ as mentioned above. As a result, the differences in tangential wind tendencies induced by total advection between $\mathrm{Vg} 3$ and CTL are quite small both above and in the inflow boundary layer (Figs. $4 \mathrm{c}$ and $5 \mathrm{c}$ ). Similar to those in CTL, the positive tangential wind tendencies in the boundary layer contributed by total advection are largely offset by the negative tendencies induced by vertical mixing including surface friction (Fig. 5d), and the tendencies due to horizontal diffusion are quite small (Fig. 5e). The net tendencies (NET, Fig. 5f) show little difference from those in CTL (Fig. 4f).

The above results can be more clearly seen from the differences in all terms in tangential wind budget between CTL and Vg3 shown in Fig. 6. The upward advection of supergradient wind component leads to large positive tangential wind tendencies in the upper part of the inflow boundary layer and immediately above (Fig. 6b), a process being considered as the boundary layer spinup mechanism of the TC eyewall above the boundary layer by Schmidt and Smith (2016) and Montgomery and Smith $(2017,2018)$. However, the positive tendencies are largely offset by the negative tendencies induced by radial advection due to outflow (Fig. 6a). As a result, the tangential wind tendencies induced by the total advection show little difference above the inflow boundary layer between $\mathrm{Vg} 3$ and $\mathrm{CTL}$ (Fig. 6c). This confirms that although the upward advection of the tangential wind from the boundary layer is responsible for the spinup of the eyewall as in Peng et al. (2018), the upward advection of the supergradient wind component has little contribution to the net tangential wind tendencies above the boundary layer, which supports the hypothesis of Heng et al. (2018). Note that the positive (negative) tendencies immediately above the 

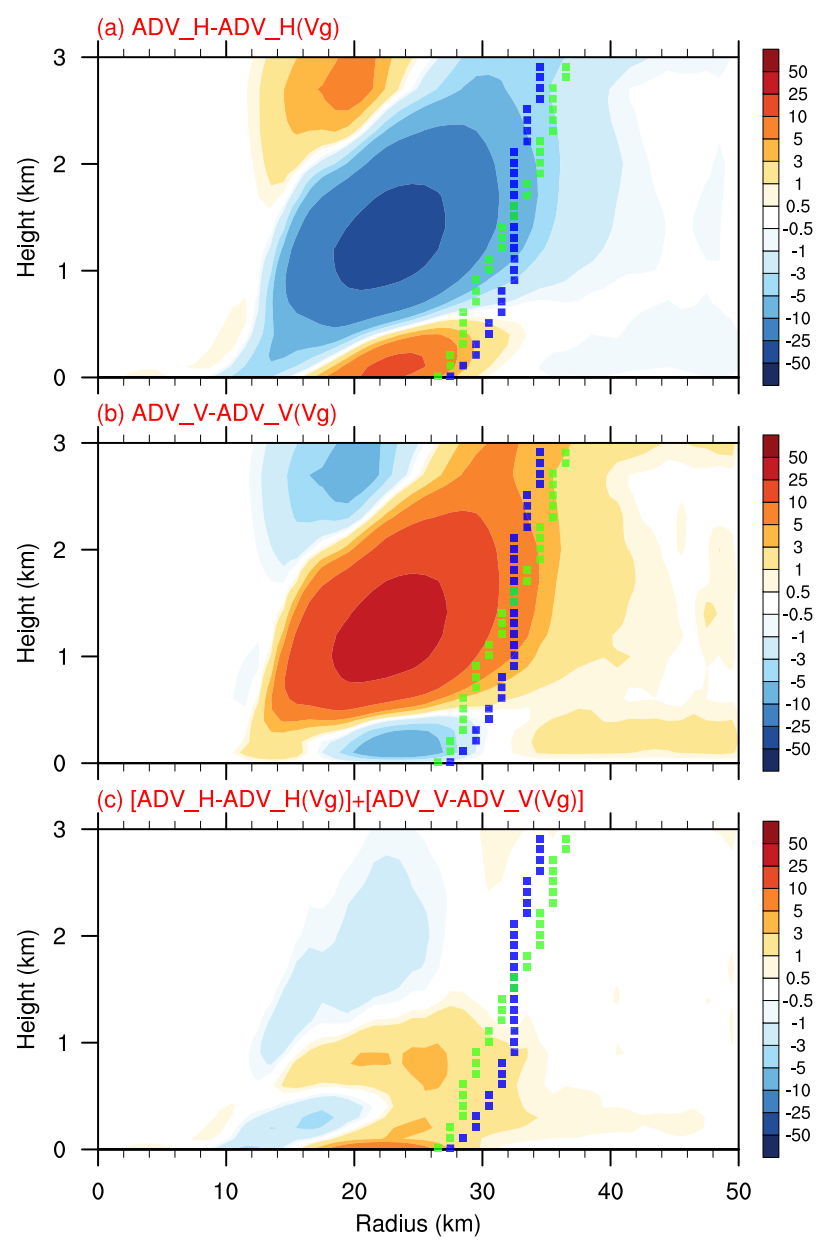
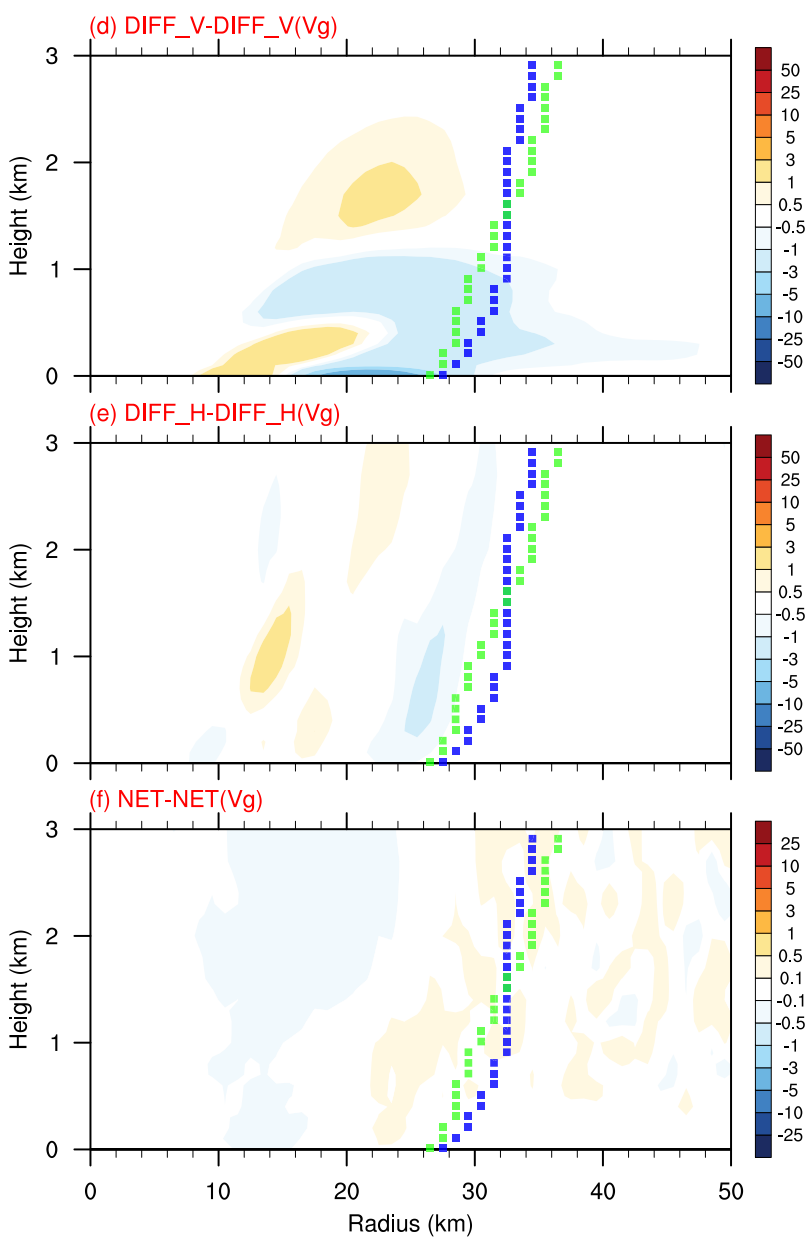

FIG. 6. As in Fig. 4, but for the differences between CTL and Vg3.

large negative (positive) tendencies induced by radial (vertical) advection in Fig. 6a (Fig. 6b) are associated with the subgradient winds and the associated inflow (Rotunno 2014). Note also that the stronger supergradient winds in the interior of the inflow boundary layer in CTL result in a larger vertical gradient of tangential winds under the eyewall than in Vg3. This leads to larger negative tangential wind tendencies in the lower part of the inflow boundary layer inside the RMW in CTL (Fig. 6b). These negative tendencies, however, are largely compensated by the positive tendencies induced by radial advection due to the relatively stronger inflow therein (Fig. 6a) as mentioned above. Some small differences in positive and negative tendencies by the total advection between Vg3 and CTL (Fig. 6c) are almost compensated by the tendencies induced by vertical mixing (Fig. 6d), which is enhanced by relatively larger vertical shear of tangential winds in CTL. The difference in the tendencies induced by horizonal diffusion between Vg3 and CTL is relatively small (Fig. 6e).
As a result, the net tangential wind tendencies show little differences in both magnitude and spatial distribution between Vg3 and CTL (Fig. 6f). This explains why the upward advection of the supergradient wind component contributes little to the intensification rate of the simulated TC.

Although the vertical advection of the supergradient wind component contributes little to the intensification rate, the storm in CTL intensified for a longer period and thus reached a higher quasi-steady intensity than that in either $\mathrm{Vg} 3$ or Vg4 (Fig. 2a). To explain the difference in the quasi-steady intensity between CTL and $\mathrm{Vg} 3$ (and $\mathrm{Vg} 4$ ), we revisited the TC maximum potential intensity (MPI) theory. According to Rousseau-Rizzi and Emanuel (2019), if the dissipative heating is not included, the theoretical MPI in terms of the maximum sustained 10-m wind speed can be given as

$$
\left|V_{10}\right|^{2}=\frac{C_{k}}{C_{D}}\left(T_{s}-T_{\text {out }}\right)\left(S^{*}-S_{10}\right),
$$



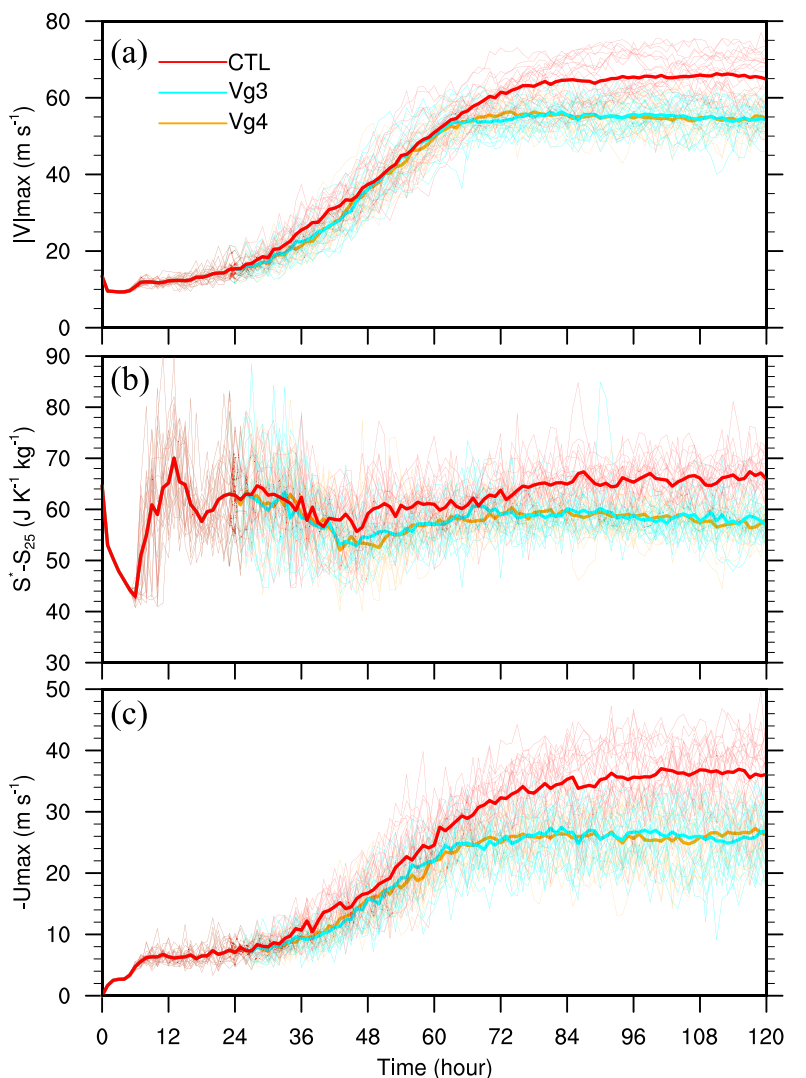

FIG. 7. As in Fig. 2, but for (a) the maximum 10-m total wind speed, (b) the difference between the surface saturated entropy at SST and the 25-m air entropy at the RMW, and (c) the maximum 10-m inflow speed.

where $\left|V_{10}\right|$ is the potential 10-m total wind speed, $T_{s}$ and $T_{\text {out }}$ are the sea surface temperature and outflow temperature in the upper troposphere, and $S^{*}$ and $S_{10}$ are the surface saturated entropy at the RMW and 10-m height air entropy at the RMW. Note that the evolutions of $10-\mathrm{m}$ total wind speed and $10-\mathrm{m}$ tangential wind speed are quite similar in each of the three experiments (Figs. 2a and 7a). Since the same ratio of surface exchange coefficients, sea surface temperature, and environmental sounding are used in all experiments, the difference in the quasi-steady intensity between in CTL and $\mathrm{Vg} 3$ (and $\mathrm{Vg} 4$ ) is most likely due to the difference in the air-sea thermodynamic disequilibrium, namely, $S^{*}-S_{10}$, in all experiments. Therefore, we compared the evolution of the air-sea thermodynamic disequilibrium at the RMW in CTL and $\mathrm{Vg} 3 / \mathrm{Vg} 4$. Note that we used the near-surface air entropy at 25-m height (at the lowest model level) instead of that at 10-m height in the comparison, as shown in Fig. 7b. The air-sea thermodynamic disequilibrium in CTL is slightly larger than that in $\mathrm{Vg} 3 / \mathrm{Vg} 4$ even with similar intensities during the primary intensification stage, indicating a potentially higher MPI of the TC in CTL than in $\mathrm{Vg} 3 / \mathrm{Vg} 4$. The difference in the air-sea thermodynamic disequilibrium between CTL and $\mathrm{Vg} 3 / \mathrm{Vg} 4$ increases with time and reached $10 \mathrm{~J} \mathrm{~K}^{-1} \mathrm{~kg}^{-1}$ in the quasi-steady stage. This explains the higher quasi-steady intensity of the storm in CTL and in $\mathrm{Vg} 3 / \mathrm{Vg} 4$. The larger air-sea thermodynamic disequilibrium in CTL is related to the stronger inflow in the inner-core region in the lower boundary layer as discussed earlier (Fig. 3), as also shown in Fig. 7c, which shows the time evolution of the maximum inflow in all experiments. The stronger inflow implies larger cold entropy advection to lower the inner-core air entropy and thus to increase the air-sea thermodynamic disequilibrium under the eyewall, as shown in the entropy budget by Rotunno and Emanuel (1987). Note that we do not attempt to give a quantitative comparison of the MPI between those experiments, because Eq. (3) assumes a local energy balance between the air-sea frictional dissipation and enthalpy flux near the RMW (Rousseau-Rizzi and Emanuel 2019), which tends to yield an underestimation of the MPI (Wang and $\mathrm{Xu}$ 2010). Based on the entropy budget, Wang and $\mathrm{Xu}$ (2010) found that the entropy flux outside about 2-2.5 times of the RMW, rather than the local $S^{*}-S_{10}$ near the RMW alone, also contributes to balance the energy dissipation near the RMW.

In addition, we also found a difference in the vertical tilt of the RMW between CTL and Vg3. Because of the larger outflow above the inflow boundary layer and the stronger inflow in the lower part of the boundary layer, the mean RMW in CTL is about $1-2 \mathrm{~km}$ smaller (larger) than that in $\mathrm{Vg} 3$ in (above) the boundary layer (Fig. 6). This leads to a relatively larger outward tilt of the RMW with height in the lower troposphere during both the primary intensification stage and the quasisteady stage in CTL than in Vg3 (Fig. 3) although the RMWs near the surface in CTL and $\mathrm{Vg} 3$ are similar (Fig. 2b). Finally, note that all those changes of structure of the $\mathrm{TC}$ in $\mathrm{Vg} 3$ from that in CTL, as mentioned above, should be regarded as a local response, mainly in the inner core and in the lower troposphere, and the overall structure of the $\mathrm{TC}$ in $\mathrm{Vg} 3$ is very similar to that in CTL (Fig. 8).

\section{Sensitivity to surface drag coefficient}

The results discussed in section 3 demonstrate that the vertical advection of the supergradient wind from the boundary layer contributes little to the intensification of the simulated TC during the primary intensification stage. Since the imbalance and the associated supergradient winds in the boundary layer are largely controlled by surface friction, which is largely determined 

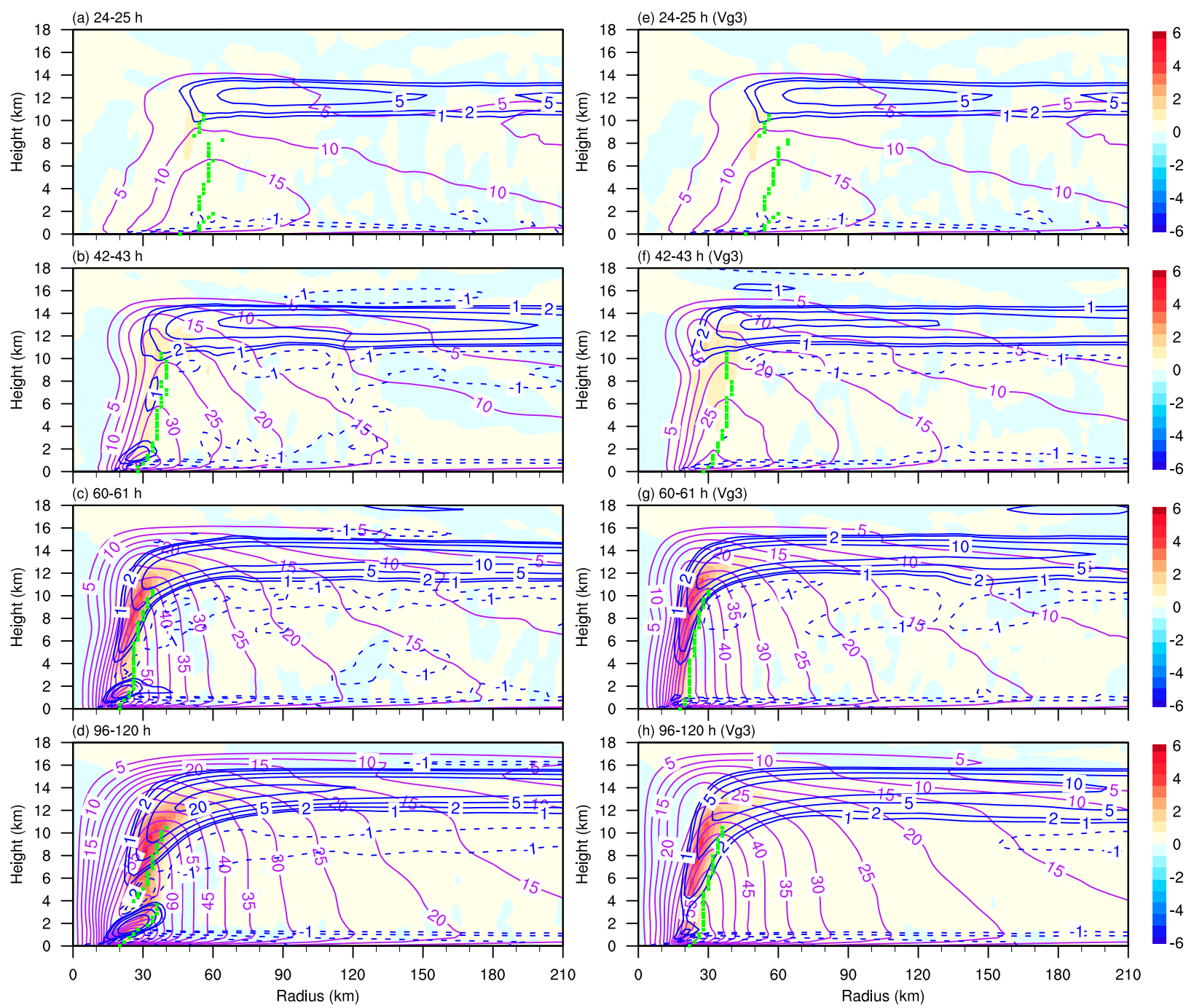

FIG. 8. The radial-vertical cross sections of the ensemble-mean vertical velocity (shading; $\mathrm{m} \mathrm{s}^{-1}$ ), tangential wind speed (purple contours; $\mathrm{m} \mathrm{s}^{-1}$ ), and the radial wind speed (blue contour with negative values dashed; $\mathrm{m} \mathrm{s}^{-1}$ ) averaged between (a) 24-25, (b) 42-43, (c) 6061, and (d) 96-120 h using model outputs at 6-min intervals from CTL. (e)-(h) As in (a)-(d), but from Vg3. The dotted green line shows the radial location of the RMW below 10-km height.

by surface drag coefficient, a natural question arises as to whether the differences between CTL and Vg3 discussed in section 3 are sensitive to surface drag coefficient. To address this issue, we have performed three additional pairs of experiments by varying the surface drag coefficient as listed in Table 1.

As we can see from Fig. 9a, although the quasisteady intensity increases with the decrease of surface drag coefficient, which is consistent with the prediction of the theoretical MPI given in Eq. (3) and the results of Peng et al. (2018, see their Fig. 11), the intensification rate during their corresponding primary intensification stages is insensitive to surface drag coefficient. Figure 10 shows the radial-height cross sections of radial winds and agradient winds averaged during their corresponding primary intensification stages (when the storms have maximum 10-m wind speed between 20 and $30 \mathrm{~m} \mathrm{~s}^{-1}$ ) in all four experiments with all default model settings. As expected, the storm with a larger surface drag coefficient developed stronger agradient winds and stronger inflow in the boundary layer and stronger outflow immediately above. However, the intensification rate of the simulated storm does not increase with the increase in the strength of supergradient winds or the upward advection of the supergradient wind component from the boundary layer, in contrast to that expected from the boundary layer spinup mechanism of TC intensification hypothesized in some previous studies. This further demonstrates that the upward advection of the supergradient wind component from 

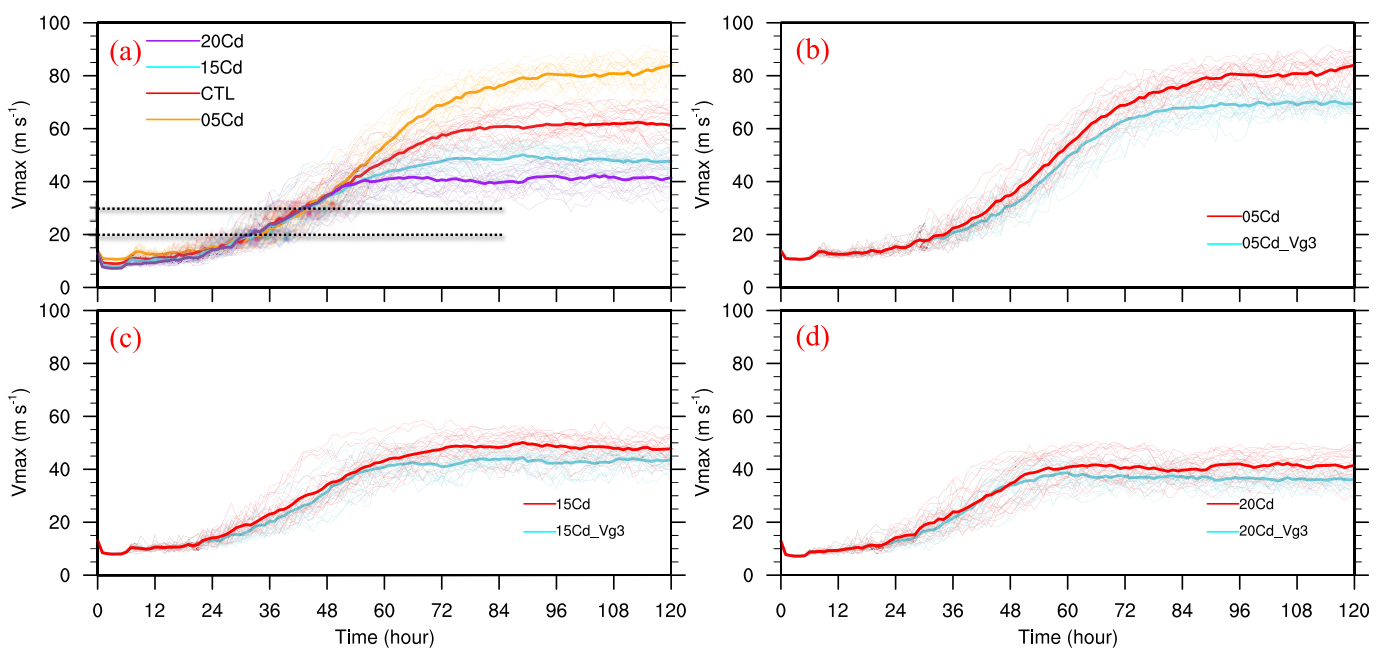

FIG. 9. Time series of the maximum 10-m tangential wind speed from different experiments with different surface drag coefficients, as indicated by the legends. The two dashed black horizontal lines in (a) mark the period for the average radial-vertical cross sections shown in Fig. 10.

the boundary layer should not be the dominant mechanism of TC intensification.

In addition, the RMW becomes smaller with larger surface drag coefficient (Fig. 10), suggesting that although changes in surface drag coefficient have little effect on the intensification rate, surface drag coefficient and thus surface friction contributes to the contraction of the simulated TC, consistent with the results of Heng and Wang (2016a). Note that the more rapid initial contraction with larger surface friction can be attributed to the larger negative radial gradient of radial advection of AAM (cf. Fig. 4a) because surface friction itself often prohibits the RMW contraction ( $\mathrm{Li}$ et al. 2019; cf. Fig. 4d).

Similar to the results discussed in section 3 , the removal of the upward advection of the supergradient wind from the boundary layer leads to a reduction of the quasi-steady intensity by $10 \%-15 \%$ but does not cause (a) $05 \mathrm{Cd}$

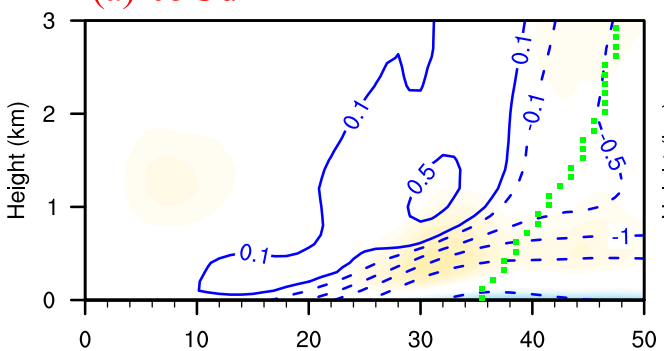

(c) $15 \mathrm{Cd}$ Radius $(\mathrm{km})$

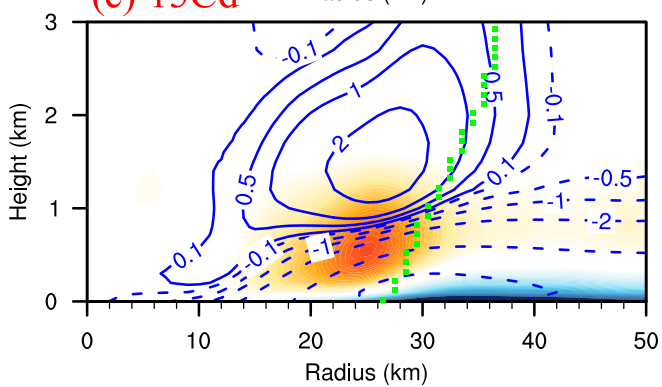

(b) CTL

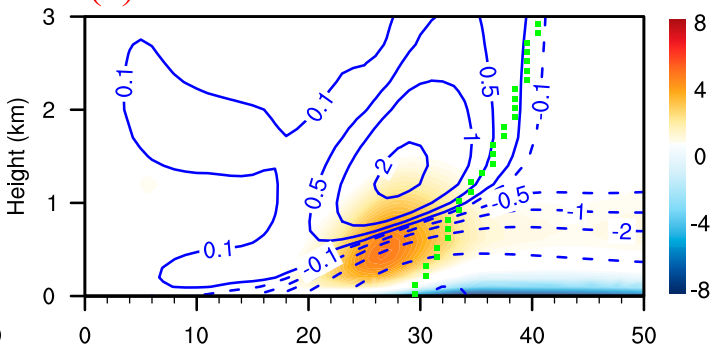

(d) $20 \mathrm{Cd}$ Radius $(\mathrm{km})$

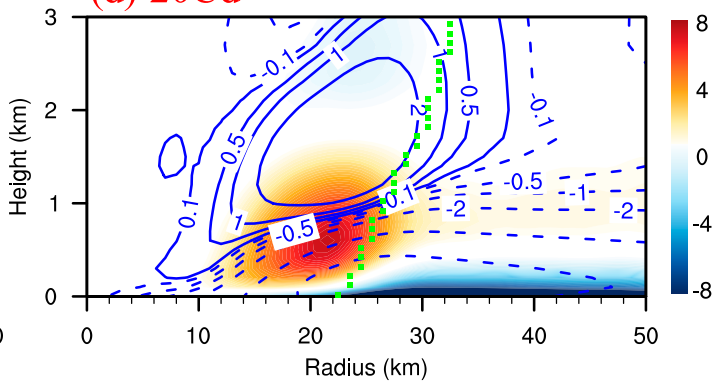

FIG. 10. The radial-vertical cross sections of the ensemble-mean radial wind (blue contours with negative values dashed; $\mathrm{m} \mathrm{s}^{-1}$ ) and agradient wind (shading; $\mathrm{m} \mathrm{s}^{-1}$ ) averaged between the two dashed horizontal lines in Fig. 9a. The RMW for each experiment is shown by the dotted green line. 
any significant change to the intensification rate of the simulated TC during their primary intensification stages in all experiments (Fig. 9). Note that a small reduction (only about $4 \%$ ) of the intensification rate during the primary intensification stage is shown in the experiment with a relatively small $C_{d}$ with the removal of the upward advection of the supergradient wind component from the boundary layer (Fig. 9b). However, this reduction should not be considered a positive contribution by the upward advection of supergradient winds from the boundary layer to the overall TC intensification rate because with this small $C_{d}$ the supergradient winds are relative weak (Fig. 10a). The small difference in the intensification rate could be caused by other changes, e.g., the relatively larger RMW (Fig. 10) in the simulations may increase the sensitivity of the intensification rate of the simulated storm to small changes in any dynamical aspects of the model. A detailed analysis is beyond the scope of this study. Nevertheless, results from these additional experiments further confirm that the upward advection of the supergradient wind contributes insignificantly to the intensification of the simulated storm during the primary intensification stage. We also have checked the radial location of the average RMW in all three additional pairs of experiments during their corresponding primary intensification period, and consistent with that in Fig. 6, the removal of the upward advection of the supergradient wind component from the boundary layer leads to a larger (smaller) mean RMW in (above) the boundary layer (not shown).

\section{Conclusions and discussion}

The existence of supergradient winds in the boundary layer is a common feature throughout the life of a TC due to the presence of surface friction. Whether supergradient winds play a dominant role in spinning up the eyewall of a TC and thus contribute to TC intensification in general is under debate. Montgomery and Smith $(2017,2018)$ proposed that the upward advection of supergradient wind from the boundary layer is a momentum source to spin up the eyewall above the boundary layer, which they called the boundary layer spinup mechanism of the TC eyewall (see also Schmidt and Smith 2016). However, Heng et al. (2018) argued that the upward advection of the supergradient wind component from the boundary layer leads to the development of an outflow layer immediately above the inflow boundary layer, which spins down the supergradient winds, and this is a gradient wind adjustment process and should not be a dominant mechanism of TC intensification. In this study, ensemble sensitivity numerical experiments using the axisymmetric TC model CM1 are performed and tangential wind budgets are conducted to quantify the net contribution by the upward advection of the supergradient wind component from the boundary layer to the intensification and final intensity of a TC.

In the control experiment all default model settings are used while in the sensitivity experiment the upward advection of the supergradient wind component from the boundary layer is artificially removed. Results from the numerical experiments show that the removal of the upward advection of the supergradient wind component from the boundary layer leads to little change to the intensification rate during the primary intensification stage (often less than $4 \%$ ) but an increase of $10 \%-15 \%$ in the quasi-steady intensity in terms of the maximum $10-\mathrm{m}$ wind speed of the simulated TC. The removal of the upward advection of the supergradient wind component from the boundary layer also largely reduces the outward agradient force and suppresses the development of an outflow layer in the inner-core region immediately above the inflow boundary layer and also reduces the supergradient winds in the boundary layer. This latter effect reduces the vertical shear of tangential wind and thus the inward agradient force and the inflow in the surface layer in the inner core. We hypothesize that it is the reduction of the inflow in the surface layer that suppresses the air-sea thermodynamic disequilibrium and thus reduces the quasi-steady intensity in the experiment with the upward advection of the supergradient wind component removed. However, we notice that the degree of thermodynamic disequilibrium does not actually change that much in time as the inflow strength greatly amplifies. Therefore, the relationship between inflow and thermodynamic disequilibrium near the surface may still need to be verified in future. We also show that these results are not sensitive to surface drag coefficient in the reasonable range we have tested. Considering that the magnitude of the supergradient jet is also strongly influenced by the vertical mixing length (e.g., Rotunno and Bryan 2012; Stern et al. 2020), an additional pair of experiments as CTL and Vg3 but using an asymptotic vertical mixing length of $100 \mathrm{~m}$ were performed (not shown), and the results are generally consistent with those discussed herein.

Results from the tangential wind budget analysis show that the upward advection of the supergradient wind component from the boundary layer indeed induces positive tangential wind tendencies in the upper part of and above the inflow boundary layer, namely, contributing positively to the spinup of the eyewall above the boundary layer as hypothesized by Montgomery and Smith $(2017,2018)$. However, the positive tendencies are largely offset by the negative tendencies induced by 
radial advection due to the resultant outflow as hypothesized by Heng et al. (2018). As a result, the net contribution by the upward advection of the supergradient wind component from the boundary layer to the tangential wind tendencies in the inner core is quite small. Therefore, the upward advection of the supergradient wind component from the boundary layer should not be a dominant mechanism of TC intensification. This is in support of the argument by Heng et al. (2018) but is in contrast with the hypothesis of Schmidt and Smith (2016) and Montgomery and Smith $(2017,2018)$. Our results thus demonstrate that it is the upward advection of high boundary layer tangential momentum associated with the gradient wind that is key to the spinup of the eyewall above the boundary layer.

Results from this study, together with previous studies of Heng et al. (2017, 2018), do not mean that the unbalanced boundary layer processes are not important to TC intensification. Rather, the unbalanced boundary layer dynamics must play key roles in controlling the strength and radial location of eyewall updraft/convection since eyewall convection in a TC is always rooted in the inflow boundary layer where mass and moisture convergence and large surface enthalpy flux are collocated. Note that some prior studies have found that TC intensification rate increases with increasing $C_{d}$ up to some threshold, e.g., $\sim 1 \times 10^{-3}$ in Peng et al. (2018, see their Fig. 11), which is lower than the minimum value in our experiments $\left(1.29 \times 10^{-3}\right)$. This means that a certain amount of surface friction is necessary for intensification of a natural TC, and as recently proposed by Kepert (2017), the unbalanced boundary layer dynamics contributes to TC intensification primarily through its control on the strength and radial location of eyewall updraft of a TC. Therefore, more efforts should be given to discover how the eyewall convection is contributed by the response of boundary layer dynamics to the TC vortex structure above the boundary layer $(\mathrm{Xu}$ and Wang 2018).

Acknowledgments. The authors thank Dr. Richard Rotunno and three other anonymous reviewers for their constructive and critical review comments. Y. Wang thanks Dr. Richard Rotunno for helpful discussions in the early stage of this work. This study has been supported in part by the National Key R\&D Program of China under Grant 2017YFC1501602 and National Natural Science Foundation of China under Grant 41730960, and in part by NSF Grant AGS-1834300. Y. Li is funded by China Scholarship Council (File 201806210324).

\section{REFERENCES}

Bryan, G. H., and J. M. Fritsch, 2002: A benchmark simulation for moist nonhydrostatic numerical model. Mon. Wea. Rev., 130, 2917-2928, https://doi.org/10.1175/1520-0493(2002)130<2917: ABSFMN $>2.0 . \mathrm{CO} ; 2$.

Bui, H. H., R. K. Smith, M. T. Montgomery, and J. Peng, 2009: Balanced and unbalanced aspects of tropical cyclone intensification. Quart. J. Roy. Meteor. Soc., 135, 1715-1731, https:// doi.org/10.1002/qj.502.

Charney, J. G., and A. Eliassen, 1964: On the growth of the hurricane depression. J. Atmos. Sci., 21, 68-75, https://doi.org/ 10.1175/1520-0469(1964)021<0068:OTGOTH $>2.0$. CO; 2 .

Dunion, J. P., 2011: Rewriting the climatology of the tropical North Atlantic and Caribbean Sea atmosphere. J. Climate, 24, 893908, https://doi.org/10.1175/2010JCLI3496.1.

Eliassen, A., 1951: Slow thermally or frictionally controlled meridional circulation in a circular vortex. Astrophys. Nor., 5, 19-60.

Emanuel, K. A., 1989: The finite-amplitude nature of tropical cyclogenesis. J. Atmos. Sci., 46, 3431-3456, https://doi.org/ 10.1175/1520-0469(1989)046<3431:TFANOT $>2.0$. CO;2.

_ 1995: The behavior of a simple hurricane model using a convective scheme based on subcloud-layer entropy equilibrium. J. Atmos. Sci., 52, 3960-3968, https://doi.org/10.1175/ 1520-0469(1995)052<3960:TBOASH>2.0.CO;2.

Heng, J., and Y. Wang, 2016a: Nonlinear response of a tropical cyclone vortex to prescribed eyewall heating with and without surface friction in TCM4: Implications for tropical cyclone intensification. J. Atmos. Sci., 73, 1315-1333, https://doi.org/ 10.1175/JAS-D-15-0164.1.

—, and — 2016b: Reply to "Comments on 'Nonlinear response of a tropical cyclone vortex to prescribed eyewall heating with and without surface friction in TCM4: Implications for tropical cyclone intensification.'” J. Atmos. Sci., 73, 5105-5109, https:// doi.org/10.1175/JAS-D-16-0262.1.

, - — , and W. Zhou, 2017: Revisiting the balanced and unbalanced aspects of tropical intensification. J. Atmos. Sci., 74, 2575-2591, https://doi.org/10.1175/JAS-D-17-0046.1.

_ — _ and —, 2018: Reply to "Comments on 'Revisiting the balanced and unbalanced aspects of tropical cyclone intensification."', J. Atmos. Sci., 75, 2497-2505, https://doi.org/ 10.1175/JAS-D-18-0020.1.

Kepert, J. D., 2017: Time and space scales in the tropical cyclone boundary layer, and the location of the eyewall updraft. J. Atmos. Sci., 74, 3305-3323, https://doi.org/10.1175/JAS-D17-0077.1.

, and Y. Wang, 2001: The dynamics of boundary layer jets within the tropical cyclone core. Part II: Nonlinear enhancement. J. Atmos. Sci., 58, 2485-2501, https://doi.org/10.1175/ 1520-0469(2001)058<2485:TDOBLJ > 2.0.CO;2.

Kessler, E., 1969: On the Distribution and Continuity of Water Substance in Atmospheric Circulation. Meteor. Monogr., No. 32, Amer. Meteor. Soc., 84 pp.

Li, Y., Y. Wang, and Y. Lin, 2019: Revisiting the dynamics of eyewall contraction of tropical cyclones. J. Atmos. Sci., 76, 3229-3245, https://doi.org/10.1175/JAS-D-19-0076.1.

Montgomery, M. T., and R. K. Smith, 2014: Paradigms for tropical cyclone intensification. Aust. Meteor. Oceanogr. J., 64, 37-66, https://doi.org/10.22499/2.6401.005.

, and _ 2017: Recent development in the fluid dynamics of the tropical cyclones. Annu. Rev. Fluid Mech., 49, 541-574, https://doi.org/10.1146/annurev-fluid-010816-060022. 
, and - 2018: Comments on "Revisiting the balanced and unbalanced aspects of tropical cyclone intensification." J. Atmos. Sci., 75, 2491-2496, https://doi.org/10.1175/JAS-D17-0323.1.

— M. E. Nicholls, T. A. Cram, and A. B. Saunders, 2006: A vortical hot tower route to tropical cyclogenesis. J. Atmos. Sci., 63, 355-386, https://doi.org/10.1175/JAS3604.1.

_ J. Persing, and R. K. Smith, 2015: Putting to rest WISHE-ful misconceptions for tropical cyclone intensification. J. Adv. Model. Earth Syst., 7, 92-109, https://doi.org/10.1002/2014MS000362.

Nguyen, S., R. Smith, and M. Montgomery, 2008: Tropical-cyclone intensification and predictability in three dimensions. Quart. J. Roy. Meteor. Soc., 134, 563-582, https://doi.org/10.1002/ qj.235.

Ooyama, K. V., 1964: A dynamical model for the study of tropical cyclone development. Geophys. Int., 4, 187-198.

, 1969: Numerical simulation of the life cycle of tropical cyclone. J. Atmos. Sci., 26, 3-40, https://doi.org/10.1175/15200469(1969)026<0003:NSOTLC $>2.0 . \mathrm{CO} ; 2$.

- 1982: Conceptual evolution of the theory and modeling of the tropical cyclone. J. Meteor. Soc. Japan, 60, 369-380, https:// doi.org/10.2151/jmsj1965.60.1_369.

Pendergrass, A. G., and H. E. Willoughby, 2009: Diabatically induced secondary flows in tropical cyclones. Part I: Quasisteady forcing. Mon. Wea. Rev., 137, 805-821, https://doi.org/ 10.1175/2008MWR2657.1.

Peng, K., R. Rotunno, and G. H. Bryan, 2018: Evaluation of a timedependent model for the intensification of tropical cyclones. J. Atmos. Sci., 75, 2125-2138, https://doi.org/10.1175/JAS-D17-0382.1.

Rotunno, R., 2014: Secondary circulations in rotating-flow boundary layers. Aust. Meteor. Oceanogr. J., 64, 27-35, https://doi.org/ 10.22499/2.6401.004.

__ , and K. A. Emanuel, 1987: An air-sea interaction theory for tropical cyclones. Part II: Evolutionary study using a nonhydrostatic axisymmetric numerical model. J. Atmos. Sci., 44, 542-561, https://doi.org/10.1175/1520-0469(1987)044<0542: AAITFT $>2.0 . \mathrm{CO} ; 2$.

, and G. H. Bryan, 2012: Effects of parameterized diffusion on simulated hurricanes. J. Atmos. Sci., 69, 2284-2299, https:// doi.org/10.1175/JAS-D-11-0204.1.

Rousseau-Rizzi, R., and K. Emanuel, 2019: An evaluation of hurricane superintensity in axisymmetric numerical models. J. Atmos. Sci., 76, 1697-1708, https://doi.org/10.1175/JAS-D18-0238.1.
Schmidt, C., and R. K. Smith, 2016: Tropical cyclone evolution in a minimal axisymmetric model revisited. Quart. J. Roy. Meteor. Soc., 142, 1505-1516, https://doi.org/10.1002/qj.2753.

Schubert, W. H., and J. J. Hack, 1982: Inertial stability and tropical cyclone development. J. Atmos. Sci., 39, 1687-1697, https:// doi.org/10.1175/1520-0469(1982)039<1687:

ISATCD $>2.0 . \mathrm{CO} ; 2$.

Shapiro, L. J., and H. E. Willoughby, 1982: The response of balanced hurricanes to local sources of heat and momentum. J. Atmos. Sci., 39, 378-394, https://doi.org/10.1175/1520-0469(1982) 039<0378:TROBHT $>2.0$. CO;2.

Smith, R. K., and M. T. Montgomery, 2015: Toward clarity on understanding tropical cyclone intensification. J. Atmos. Sci., 72, 3020-3031, https://doi.org/10.1175/JAS-D-15-0017.1.

— and —, 2016: Comments on "Nonlinear response of a tropical cyclone vortex to prescribed eyewall heating with and without surface friction in TCM4: Implications for tropical cyclone intensification." J. Atmos. Sci., 73, 5101-5103, https:// doi.org/10.1175/JAS-D-16-0163.1.

— - , and N. V. Sang, 2009: Tropical cyclone spin-up revisited. Quart. J. Roy. Meteor. Soc., 135, 1321-1335, https:// doi.org/10.1002/qj.428.

Stern, D. P., J. L. Vigh, D. S. Nolan, and F. Zhang, 2015: Revisiting the relationship between eyewall contraction and intensification. J. Atmos. Sci., 72, 1283-1306, https://doi.org/10.1175/ JAS-D-14-0261.1.

_ J. J. Kepert, G. H. Bryan, and J. D. Doyle, 2020: Understanding atypical midlevel wind speed maxima in hurricane eyewalls. J. Atmos. Sci., 77, 1531-1557, https://doi.org/10.1175/JAS-D-190191.1.

Wang, Y., and J. Xu, 2010: Energy production, frictional dissipation, and maximum intensity of a numerically simulated tropical cyclone. J. Atmos. Sci., 67, 97-116, https://doi.org/ 10.1175/2009JAS3143.1.

Willoughby, H. E., 1979: Forced secondary circulations in hurricanes. J. Geophys. Res., 84, 3173-3183, https://doi.org/10.1029/ JC084iC06p03173.

Wood, V. T., and L. W. White, 2011: A new parametric model of vortex tangential-wind profiles: Development, testing, and verification. J. Atmos. Sci., 68, 990-1006, https://doi.org/ 10.1175/2011JAS3588.1.

$\mathrm{Xu}$, J., and Y. Wang, 2018: Effect of the initial vortex structure on intensification of a numerically simulated tropical cyclone. J. Meteor. Soc. Japan, 96, 111-126, https://doi.org/10.2151/ jmsj.2018-014. 